\title{
Comparison of Fingerprint Minutiae Matching Technologies
}

\author{
B.Sudeepthi ${ }^{1}$, Md.Imaduddin ${ }^{2}$, D.Kavitha ${ }^{3}$ \\ ${ }^{I}$ MTech Scholar, Aurora's Scientific Technological and Research Academy, Hyderabad, TS, India. \\ ${ }^{2}$ Associate Professor, Dept of ECE. Aurora's Scientific Technological and Research Academy, Hyderabad. \\ ${ }^{3}$ Associate Professor, Dept of ECE. Aurora's Scientific Technological and Research Academy, Hyderabad.
}

\begin{abstract}
Now a day's recognition of persons is performed by using biometric technologies like facial recognition, fingerprint recognition, voice recognition, iris recognition and hand geometry. Among all these recognition technologies fingerprint recognition is most popular technique because of its simplicity. Fingerprint technology was simple because of two reasons. First one, it requires very less effort from the person, does not require other information than necessary for the recognition process and provides relatively good performance. Second one, the cost of fingerprint sensors was relatively less which enables easy integration into wireless hardware like PC keyboards. Fingerprint is the combination of ridges and valleys. There are two types of fingerprint matching technologies, namely correlation based and minutiae based. Between these two technologies minutiae based matching is most widely used one. In fingerprint terms, minutiae point is defined as ridge ending point or ridge bifurcating point. Ridge ending is the point at which the ridge ends abruptly and ridge bifurcation is the point at which the ridge divides into two. This means each fingerprint consists of a number of minutiae points and the combination of minutiae points is known as minutiae descriptor. Each minutiae is represented by the particular properties like orientation, location and minutiae type(whether ridge ending or ridge bifurcating). Decision is made on the match between a pair of minutiae depending on the similarity of these properties. This paper focus on the three types of minutiae matching technologies, named Jiang, Novel and Modified.

Keywords: Minutiae, similarity level and matching score
\end{abstract}

\section{Introduction}

Among all biometric traits, fingerprints have one of the highest levels of reliability and have been extensively used by forensic experts in criminal investigations. A fingerprint refers to the flow of ridge patterns in the tip of the finger. The ridge flow exhibits anomalies in local regions of the fingertip and it is the position and orientation of these anomalies that are used to represent and match fingerprints.

The uniqueness of a fingerprint is determined by the topographic relief of its ridge structure and the presence of certain ridge anomalies termed as minutiae points. Typically, the global configuration defined by the ridge structure is used to determine the class of the fingerprint, while the distribution of minutiae points is used to match and establish the similarity between two fingerprints. Automatic fingerprint identification systems, that match a query print against a large database of prints (which can consist of millions of prints), rely on the pattern of ridges in the query image to narrow their search in the database (fingerprint indexing), and on the minutiae points to determine an exact match (fingerprint matching). The ridge flow pattern itself is rarely used for matching fingerprints.

Minutiae, in fingerprinting terms, are the points of interest in a fingerprint, such as ridge bifurcations and ridge endings.

Examples are: ridge endings - a ridge that ends abruptly, ridge bifurcation - a single ridge that divides into two ridges, short ridges or independent ridge - a ridge that commences, travels a short distance and then ends, ridge enclosures - a single ridge that bifurcates and reunites shortly afterward to continue as a single ridge, spur - a bifurcation with a short ridge branching off a longer ridge, crossover or bridge - a short ridge that runs between two parallel ridges

The fig 1 illustrates the types of minutiae. There are many types of minutiae but the focus when matching is only on the two main minutiae, ridge ending and ridge bifurcation. When a ridge ends abruptly, that point is known as ridge ending or ridge termination and when a single divides into two, that point is known as ridge bifurcation. 


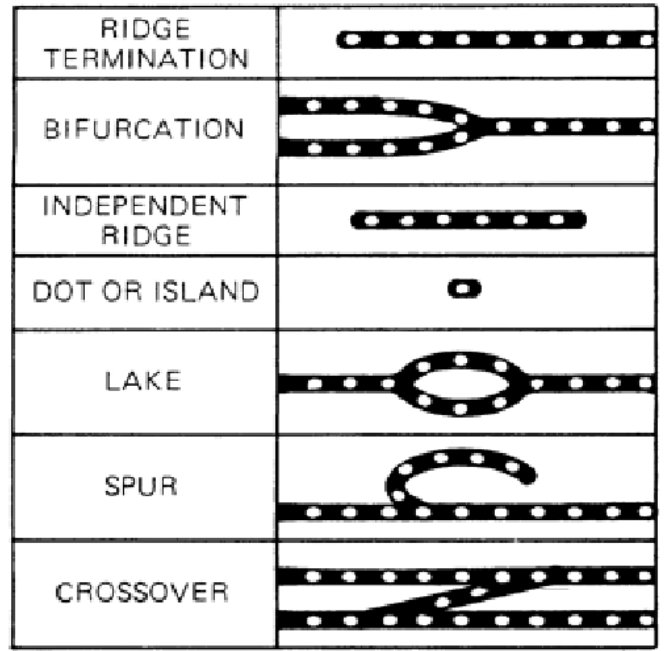

Fig 1. Minutiae types

\section{General Identification System}

There are two types variations in fingerprint matching, large intra-class(variations among different impressions of the same finger) and small inter-class variations(images of different fingers may appear quite similar). Fingerprint matching is a difficult problem due to these variations. Three fundamental reasons for the large intra-class variations are partial overlap, non-linear distortion, and sensor noise. Due to rotation and displacement of the finger placed on the sensor, there is often only a partial overlap between the template and query fingerprint images. This problem is more severe in the case of small solid-state sensors that are being increasingly deployed. The mapping of a three-dimensional finger into a two-dimensional image results in nonlinear distortion. Further, changes in the applied finger pressure and skin conditions (dry skin, sweat, etc.) cause a change in the thickness of the ridges. Noise introduced in the fingerprint sensor in the form of residues left over the sensor surface due to repeated usage also contributes to the intra-class variations. Although it is unlikely that impressions of different fingers are identical in all respects, they may be quite similar in terms of their global structure and ridge orientations. This can lead to a number of false matches when the matchers rely on global features alone. The results of the Fingerprint Verification Competition 2004 (FVC2004) show that even the state-of-the-art fingerprint matchers do not achieve extremely high accuracy when the database contains images of poor quality. The best (commercial) fingerprint matcher in FVC2004 had an equal error rate (EER) of $2.07 \%$ indicating that fingerprint matching remains a challenging task in the field of pattern recognition and image processing.

Fig 2 represents a general representation of fingerprint identification system. Fingerprint matching is the last step in fingerprint identification system.

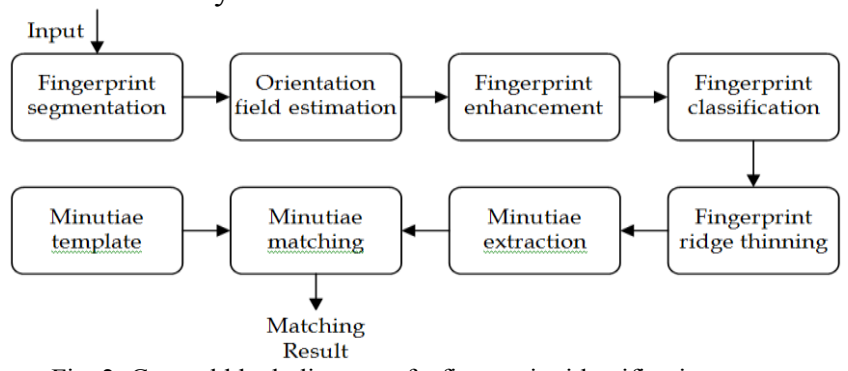

Fig 2. General block diagram of a fingerprint identification system

\section{System Level Design}

There are three main stages in the fingerprint recognition process. First one is the acquisition of fingerprints, second one is marking of minutiae points and final stage is the matching of minutiae.

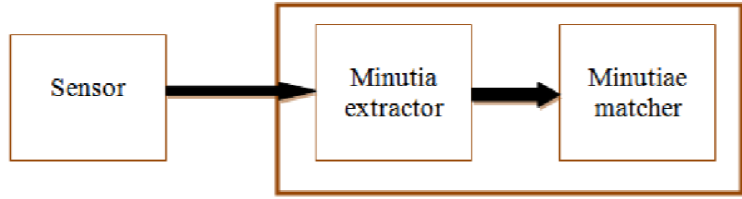

Fig 3. Fingerprint recognition system 
Optical or semiconductor sensors are used for fingerprint acquisition. These sensors have good efficiency and acceptable accuracy except for some cases that the user's finger is too dirty or dry. However, the testing database for my project consists of scanned fingerprints of FVC2002 DB1(that is fingerprint images of FVC2002's databse1).

As the name indicates minutiae extractor stage is used to mark the minutiae points. Minutiae extraction is done in three stages image preprocessing, minutiae marking and post-processing as shown in figure4.

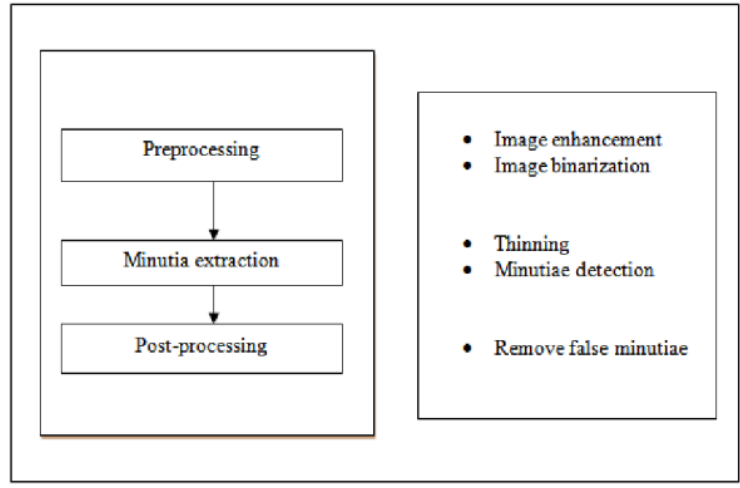

Fig 4. Minutiae extractor

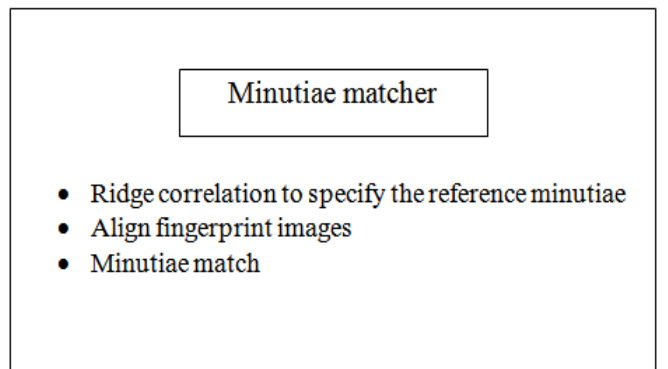

Fig 5. Minutiae matcher

Again there are two sages in preprocessing, image enhancement and image binarization. Enhancement of image is required for make the image clear for further processing. Binarization is the process of converting gray scale image into a binary image. Histogram Equalization and Fourier Transform are used for image enhancement. And locally adaptive threshold method is used for image binarization. The minutia marking is a relatively simple task. For minutia extraction stage, iterative parallel thinning algorithm is used.

For the post-processing stage, a more rigorous algorithm is developed to remove false minutia. The minutia matcher chooses any two minutiae as a reference minutia pair and then matches their associated ridges first. If the ridges match well, the two fingerprint images are aligned and matching is conducted for all the remaining minutiae.

\section{Proposed Algorithm}

The fig 6 illustrates the process involved in my project and these steps are discussed below in detail.

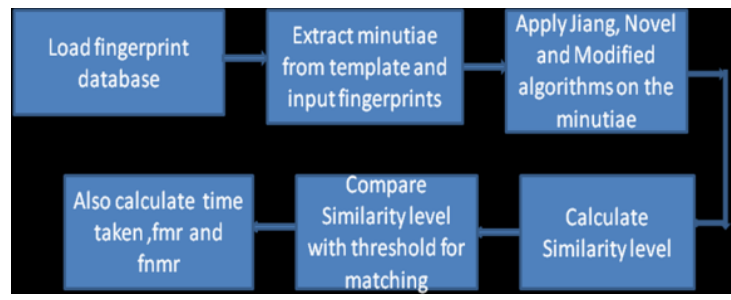

Fig 6.Block diagram of fingerprint matching algorithm

- Initially the fingerprint database FVC2002 DB1 is loaded with 72 Images. Each of the fingerprint is classified to be input fingerprint whose minutiae are extracted.

- The template fingerprint is read and its corresponding minutiae are extracted.

- Before the minutiae are extracted the fingerprint is subjected to the following operations: Enhancement, making mask, filtering minutiae and filtering false minutiae.

- Extracted minutiae consist of the corresponding coordinates of the ridge, it's direction and angle.

- Initially comparison is done using Jiang algorithm. The steps are given below 


\section{A. Jiang algorithm}

- Initially comparison is done using Jiang algorithm. The steps are given below

$>$ The Euclidean distance between each of the pairs of input and template fingerprints is calculated.

$>$ The average angle is also calculated.

$>$ Similarity level is then calculated by choosing weights of each of the feature vectors and applying respective equations.

$$
\begin{aligned}
& \mathrm{sl}(\mathrm{i}, \mathrm{j})=\left\{\begin{array}{cc}
\frac{\mathrm{bl}-\mathbf{W}\left|\mathrm{Fl}_{\mathrm{i}}^{\mathrm{I}}-\mathrm{Fl}_{\mathrm{j}}^{\mathrm{T}}\right|}{\mathrm{bl},}, & \text { If } \mathbf{W}\left|\mathrm{Fl}_{\mathrm{i}}^{\mathrm{I}}-\mathrm{Fl}_{\mathrm{j}}^{\mathrm{T}}\right|<\mathrm{bl} \\
0, & \text { otherwise }
\end{array}\right. \\
& W=\left(w_{d} w_{d} w_{\theta} w_{\theta} w_{\varphi} w_{\varphi} w_{n} w_{n} w_{t} w_{t} w_{t}\right)
\end{aligned}
$$

$>$ The row number and column number where the maximum similarity level occurs is retrieved.

$>$ Using the above obtained values the corresponding feature vectors are obtained.

$>$ Due to Non linear deformation and false minutiae matching, global structures is used for final alignment.

$>$ A Bounding box is taken with parameters $\left[\begin{array}{lll}10 & 16 & 10\end{array}\right]$ and similarity level is computed using the below equations

$$
\mathrm{Fg}_{\mathrm{k}}=\left(\begin{array}{c}
\mathrm{r}_{\mathrm{bk}} \\
\varphi_{\mathrm{bk}} \\
\theta_{\mathrm{bk}}
\end{array}\right)=\left\{\begin{array}{c}
\sqrt{\left(\mathrm{x}_{\mathrm{b}}-\mathrm{x}_{\mathrm{k}}\right)^{2}+\left(\mathrm{y}_{\mathrm{b}}-\mathrm{y}_{\mathrm{k}}\right)^{2}} \\
\mathrm{~d} \phi \mid\left(\tan ^{-1}\left(\frac{\mathrm{y}_{\mathrm{b}}-\mathrm{y}_{\mathrm{k}}}{\mathrm{x}_{\mathrm{b}}-\mathrm{x}_{\mathrm{k}}}\right), \theta_{\mathrm{b}}\right) \\
\mathrm{d} \phi\left(\theta_{\mathrm{h}}, \theta_{\mathrm{k}}\right)
\end{array}\right\}
$$

The final matching score is computed using

$$
\text { Ms }=100 \times \frac{\sum_{i, j} \operatorname{ml}(\mathrm{i}, \mathrm{j})}{\max \{\mathrm{M}, \mathrm{N}\}}
$$

\section{B. Novel algorithm}

- Due to more time and more no of computations, a Novel algorithm is proposed which is an improvement over Jiang algorithm.

> For each minutiae $\mathrm{M}_{i},{ }^{\mathrm{M}_{j}}$ from the input and template fingerprint, we create their local structures $\mathrm{Fl}_{\mathrm{i}}^{\mathrm{I}}$ and $\mathrm{Fl}_{\mathrm{j}}^{\mathrm{T}}$

For each pair of local structure, we calculate its similarity value using Jiang algorithm equation

$>$ We eliminate all pairs of local structures that have a similarity value less than LocalScoreTHr (new threshold). Only the principal minutiae for each pair of local structures is retained.

$>$ We sort all minutiae pairs selected in local matching phase descendent order according to their similarity value.

$>$ We align all minutiae of input fingerprint and validate them using the Boundary box parameters.

$>$ Finally, we compute the final matching score Ms.

\section{Modified algorithm}

- To further improve the algorithm in terms of correct algorithm, we propose a modified algorithm for fingerprint matching

$>$ After extracting all the local features of both the input and template fingerprint, they are transformed to their corresponding polar coordinate system.

$>$ The Euclidean distance vector and theta vector are then compared with the threshold values and every time a variable is incremented if it is below the threshold level.

$>$ Finally the Similarity index is calculated using the Equation

$\mathrm{S}=\operatorname{sqrt}\left(\mathrm{n}^{\wedge} 2 / \mathrm{C} 1 * \mathrm{C} 2\right)$

$\mathrm{n}$ : variable that is incremented.

$\mathrm{C} 1$ : Minutiae of input

$\mathrm{C} 2$ : Minutiae of template

- All the Similarity levels obtained by the 3 algorithms are compared with a threshold value to conclude which input fingerprint matched with the template fingerprint.

- The matched fingerprint numbers are displayed.

- The time elapsed for each of the 3 techniques is also displayed. False Matching rate (FMR) and False Non matching rate (FNMR) is also calculated and displayed. 


\section{Results}

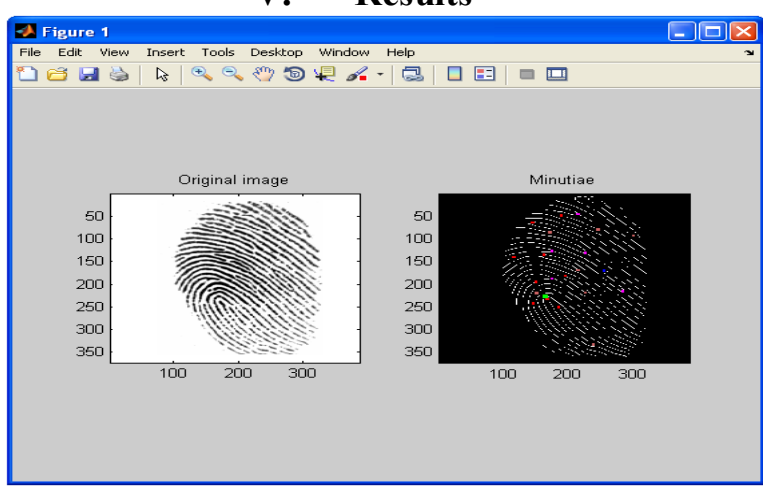

Fig 7.Fingerprint minutiae marking

Matched_FingerPrints_Jiang =

1

21

36

42

44

54

6.5

Fig 8. Matched fingerprints Jiang

Matched
1
3
10
11
19
22
23
24
25
26
28
31
33
43
45
47
48
49
50
56
61
66
67
69
71

Fig 9. Matched fingerprints Novel

\section{Matched_FingerPrints_ext $=$}

$\begin{array}{llllllll}1 & 2 & 3 & 4 & 5 & 6 & 7 & 8\end{array}$

Fig 10. Matched fingerprints modified 


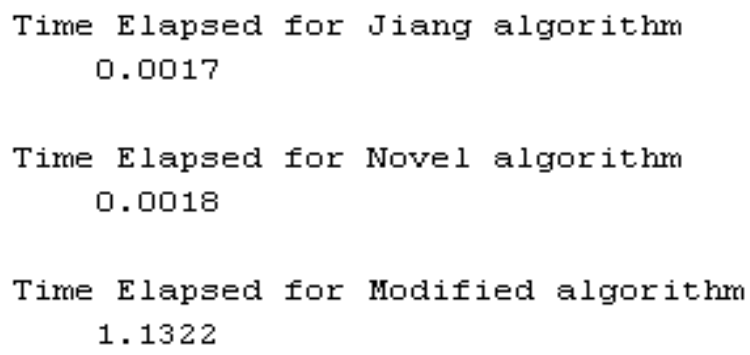

Fig 12. FMR and FNMR

\section{Conclusion}

For an efficient minutiae matching algorithm fimr should be low and fnmr should be high. This can be achieved by using Modified algorithm.

\section{Reference}

[1]. K. Jain, L. Hong, R. Bolle, “On-line fingerprint verification,” IEEE Trans. Pattern Analysis and Machine Intelligence, vol. 19, no. 4, 1997.

[2]. Cappelli, R., Maio, D., Maltoni, D., Wayman, J.L., Jain, A.K.: performance evaluation of fingerprint verification systems. IEEE Trans. Pattern Anal. Mach. Intell. 28, pp. 3-18, 2006.

[3]. Feng, J.: Combining minutiae descriptors for fingerprint matching. Pattern Recognit. 41, pp. 342-352, 2008.

[4]. Feng, J., Ouyang, Z., Cai, A.: Fingerprint matching using ridges. Pattern Recognit. 39, pp. 2131-2140, 2006.

[5]. Wang, X., Li, J., Niu, Y.: Fingerprint matching using Orientation Codes and PolyLines. Pattern Recognit. 40, 3164-3177, 2007.

\section{About the authors:}

B.Sudeepthi received B.Tech Degree in ECE from SVSSIT, Warangal, Telangana in 2012. Pursuing M.Tech in ECE at Aurora's Scientific, Technological and Research Academy,(Affiliated to JNTU) Hyderabad, Telangana.

Md.Imaduddin, Associate Prof, ECE Dept, Aurora's Scientific, Technological and Research Academy, Hyderabad, Telangana. B.Tech in ECE from JNTU Hyderabad. M.Tech in Digital electronics and computer systems from JNTU Anantapur. Area of interest Digital Signal processing, Digital image processing.

D.Kavitha, Associate Prof, ECE Dept, Aurora's Scientific, Technological and Research Acadaemy, Hyderabad, Telangana. BE in ECE from Maturi Venkata Subbarao (OU), Hyderabad. M.Tech in VLSI from Gokaraju Rangaraju Institute of Engineering and Technology, Hyderabad. 